\title{
Laser-induced ultrasonic measurements for the detection and reconstruction of surface defects
}

\author{
Feiming Qian ${ }^{1}$, Guangzhen Xing ${ }^{2}$, Ping Yang ${ }^{2}$, Pengcheng $\mathrm{Hu}^{1}$, Limin Zou ${ }^{1, *}$, and Triantafillos Koukoulas ${ }^{3}$ \\ ${ }^{1}$ Institute of Ultra-Precision Optoelectronic Instrument Engineering, Harbin Institute of Technology, Harbin 150080, PR China \\ ${ }^{2}$ Division of Mechanics and Acoustics, National Institute of Metrology, Beijing 100029, PR China \\ ${ }^{3}$ Metrology Research Centre, Mass and Related Quantities Team, Acoustics, Ultrasound and Vibration Metrology, \\ National Research Council Canada, 1200 Montreal Road, Ottawa, Ontario K1A 0R6, Canada
}

Received 28 June 2021, Accepted 13 August 2021

\begin{abstract}
Laser-induced ultrasonic measurement is a non-contact non-destructive technology that can be employed for the testing and assessment of surface defects. In order to improve the correct identification of defects, the full matrix capture (FMC) and total focusing method (TFM) are applied on the imaging process. FMC data includes A-scans resulting from the combination of all measurement axes defined by the sequential generation and detection of utilized laser beams in the system. In this paper, an aluminium block with four holes whose diameters range from $1 \mathrm{~mm}$ to $2.5 \mathrm{~mm}$ is assessed through B-scans, the synthetic aperture focusing technique (SAFT) and FMC/TFM. The results demonstrate that the FMC/TFM technology can significantly improve the imaging quality and signal-to-noise ratio (SNR). In addition, this method has higher lateral resolution and larger imaging range compared with traditional B-scans.
\end{abstract}

Keywords: Laser-induced ultrasound, Surface defects detection, Full matrix capture, Total focusing method

\section{Introduction}

Surface defects are the most common damage forms in materials, such as fatigue cracks on rail surfaces and micro-cracks on the surfaces of metal containers. These defects are shallow and small in size at the beginning (generally less than $1 \mathrm{~mm}$ ) and are not easy to detect, but will gradually deepen and become larger with usage, potentially causing serious accidents. Therefore, the detection of such surface defects is of great significance in aviation, aerospace and machinery industries amongst other fields [1, 2]. Rayleigh waves propagate along the surface with low attenuation and their energy is manly concentrated within the depth of one wavelength, thus giving the possibility of scanning a large area at a time [3, 4]. This technique is suitable for the detection of surface defects and is more accurate than penetrant testing using liquids or dyes and safer compared to radiographic testing. As a result, Rayleigh waves offer the potential for the detection of surface and subsurface defects. When Rayleigh waves interact with defects, part of the propagated waves will be transmitted and reflected, that is subsequently used for the defects visualization and depth analysis [5].

Traditional surface defect detection techniques mainly use monolithic wedge transducers to induce and receive

*Corresponding author: zoulimin@hit.edu.cn
Rayleigh waves. The transducers have a large beam profile that limits the accuracy for small defect detection and significantly limits the off-axis detection capabilities of defects due to the low signal-to-noise ratio (SNR). In view of these limitations, array transducers have been used with a wedge whose angle is equal to the third critical angle to improve the sensitivity and range of detection, but this method limits the imaging area directly below the wedge and requires a series of time delays to realize the deflection of the acoustic beam [6]. In recent years, the full matrix capture (FMC) using total focusing method (TFM) has been applied to accurately characterize surface defects as shown in Figure 1, in addition to imaging outside the wedge area without complex control [7, 8]. However, all the methods require simple geometry wedge-coupled samples and have significant limitation in detecting complex structures. Additionally, the attenuation within the wedge and the leaky loss of Rayleigh waves impose further detection limitations especially for high frequency signals [9].

In order to overcome the problems mentioned above, laser-induced ultrasound can be used for surface defect detection. For applications in non-destructive evaluation, ultrasonic waves are generated by laser irradiation based on the thermo-elastic effect, while laser interferometry is used for detection. Compared to traditional piezoelectric and electrical vibration measurement methods, there is no requirement to use wedges, which in turn facilitates large 
area scanning and avoids the limitations imposed due to high frequency signals. Moreover, laser-induced ultrasound can detect vibration signals in many harsh environments, such as high temperature, pressure and radiation as well as strong corrosion $[10,11]$. Nonetheless, the detection efficiency and robustness are still the main obstacles for wide application of laser-induced ultrasonic technology. In view of this, strategies of laser-induced ultrasonic phased array technology have been proposed. Some use multiple optical fibers with variable length to generate phased-array laser beams [12], others synthesizes laser ultrasonic array to image the internal defects $[13,14]$. However, most of the research of laser-induced ultrasonic phased array technology has been limited to longitudinal waves, to the authors' knowledge, this technique has not been used for surface defect detection.

In this paper, we present laser-induced ultrasonic phased array measurements based on FMC/TFM technology for the assessment of surface defects by the usage of Rayleigh waves. The utilization of a laser-induced ultrasonic phased array technology avoided the effect of coupling on detection, a major deficiency of traditional array detection. In addition, the synthetic aperture focusing technique (SAFT) combined with B-scans has also been used to surface flaw imaging. First, the principles of FMC/TFM using a laser-induced ultrasonic array to image surface defects are described. Then, we introduce the experimental design and the results from an aluminium block with drilled holes whose diameters range from $1 \mathrm{~mm}$ to $2.5 \mathrm{~mm}$. Finally, the discussion and conclusions are presented. The usage of laser-induced ultrasonic array and FMC/TFM imaging technology offers a good solution for surface defect detection, and may reach a wide application in non-destructive area.

\section{The principles of FMC/TFM}

Similar to SAFT, FMC/TFM utilizes synthetic apertures to improve the detection resolution and the system SNR. The key difference is on the data acquisition approach. To be specific, FMC data includes A-scans from all the transmitted and received components obtained by the sequential excitation and response of the array elements. However, SAFT data is acquired by the generation and detection of each element which is equivalent to B-scans. Thus, the FMC/TFM method may be regarded as an improvement of the SAFT approach.

Assuming that the number of array elements is $N$, the FMC can be described as follows. Full matrix data acquisition includes ultrasonic emissions of $N$ elements, where each is sequentially excited as shown in Figure 2a. When the ith element generates an ultrasonic signal, all the elements receive the A-scan signals of echo data $S_{i j}(i=1,2, \ldots$, $N ; j=1,2, \ldots, N)$ simultaneously, so the full matrix data $\left\{S_{i j}\right\}$ of $N \times N$ can be obtained as shown in Figure $2 \mathrm{~b}$. The advantages of FMC is that most conventional phased array ultrasonic detections can be achieved by applying specific post-processing algorithms to matrix data.

In order to adapt FMC for laser-induced ultrasonic array, the laser is moved to induce Rayleigh waves in all

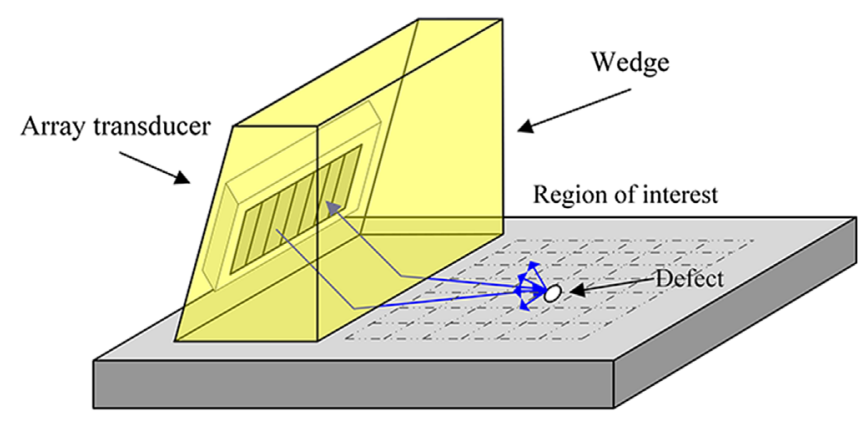

Figure 1. Schematic view of surface defects imaging based on FMC/TFM using array transducers with a wedge.

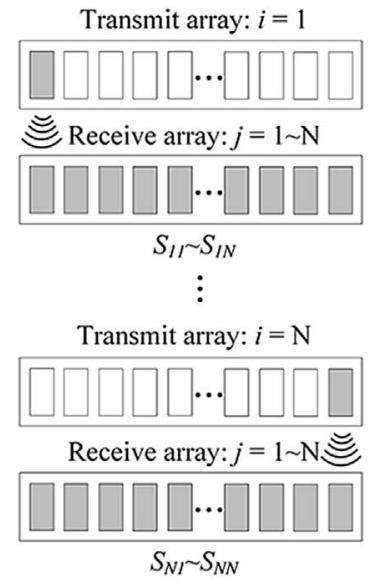

(a)

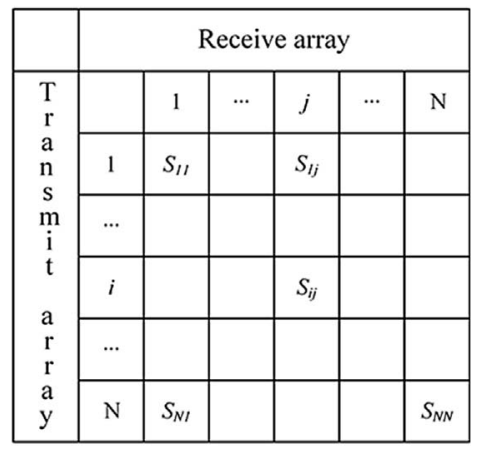

(b)
Figure 2. (a) The principle of FMC. Every element is excited sequentially, while all elements receiving A-scan signals of the echo data. (b) The full matrix data $\left\{S_{i j}\right\}$ of $N \times N$.

directions at the array positions in sequence. When a surface defect is encountered along the propagation path, Rayleigh waves will scatter and the detection interferometer is used to scan across array positions along $x$-axis to collect scattering signals. Following, the full matrix of $N \times N$ can be acquired, where $N$ is the number of array positions, as described in Figure 3, and the data of $S_{j j}(j=1,2, \ldots, N)$ can be used for SAFT imaging.

Unlike phased arrays, the delay or focus scheme is not required in FMC, instead the image reconstruction algorithm is used for total focusing. The TFM is considered as the reference standard in phased array imaging [15]. For full-matrix imaging, the region of interest is identified and then discretized into grid points $P_{i}\left(x_{p}, y_{p}\right)$ at an appropriate resolution, for instance half of the acoustic wavelength. The amplitude of sound pressure at any point in the region of interest is considered as the contribution of acoustic waves transmitted by different array elements. To be specific, the acoustic amplitude $A\left(x_{p}, y_{p}\right)$ at point $P_{i}\left(x_{p}, y_{p}\right)$ can be expressed by:

$$
A\left(x_{p}, y_{p}\right)=\sum_{i=1}^{N} \sum_{j=1}^{N} S_{i j}\left[t_{i j}\left(x_{p}, y_{p}\right)\right]
$$




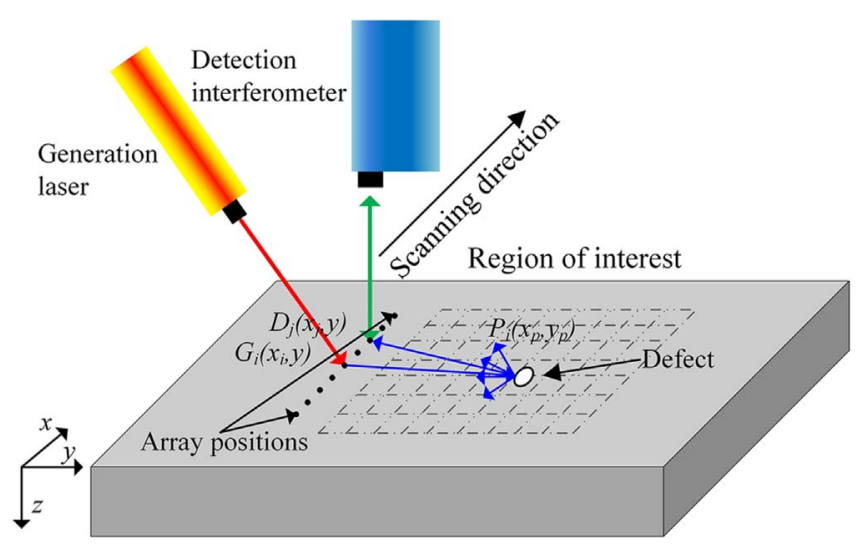

Figure 3. Schematic diagram of laser-induced ultrasonic for FMC data acquisition. The ultrasonic signals are generated by a pulsed laser and the interferometer scans across all positions parallel to the $x$-axis. Then, the pulse laser is moved to the next position while the interferometer is used to scan repeatedly.

where $t_{i j}$ is the propagation time of Rayleigh waves from element $i$ through point $P_{i}\left(x_{p}, y_{p}\right)$ to element $j$ and $t_{i j}\left(x_{p}, y_{p}\right)$ is given by:

$$
\begin{aligned}
& t_{i j}\left(x_{p}, y_{p}\right)= \\
& \frac{\sqrt{\left(x_{i}-x_{p}\right)^{2}+\left(y-y_{p}\right)^{2}}+\sqrt{\left(x_{j}-x_{p}\right)^{2}+\left(y-y_{p}\right)^{2}}}{c_{R}} .
\end{aligned}
$$

It may be seen from Figure 3 that $G_{i}\left(x_{i}, y\right)$ is the $i$ th emission position, and $D_{j}\left(x_{j}, y\right)$ is the $j$ th detection position; Equation (1) represents the acoustic pressure at point $P_{i}\left(x_{p}, y_{p}\right)$. Therefore, the amplitude at each point in the region of interest can be obtained to image any surface defects present.

\section{Experiment and discussion}

\subsection{Rayleigh wave experiment}

The aluminum block used for laser-induced ultrasonic measurements is a thin plate. Rayleigh or Lamb waves can be generated in the plate, while the velocity of these waves being similar. In order to verify what kind of waves are measured, a wedge aluminum block with different thickness was constructed. The schematic diagram of the testing block is shown in Figure 4.

The utilized laser was an infrared Nd:YAG (INNOLAS SpitLight 500) generating $8 \mathrm{~ns}$ duration pulses at $1064 \mathrm{~nm}$ with a repetition rate of $20 \mathrm{~Hz}$ and an energy of approximately $100 \mathrm{~mJ}$. The spot size of laser is $6 \mathrm{~mm}$ and an adjustable aperture was used for adjusting the diameter of the laser beam in order to change the laser energy. After being focused by a convex lens, the laser was directed towards the surface of sample and induced ultrasonic signals along the $y$ direction, which were detected by a two-wave mixing interferometer (Bossa Nova TEMPO-2D) operating at $532 \mathrm{~nm}$. The detecting beam was focused to a $75 \mu \mathrm{m}$

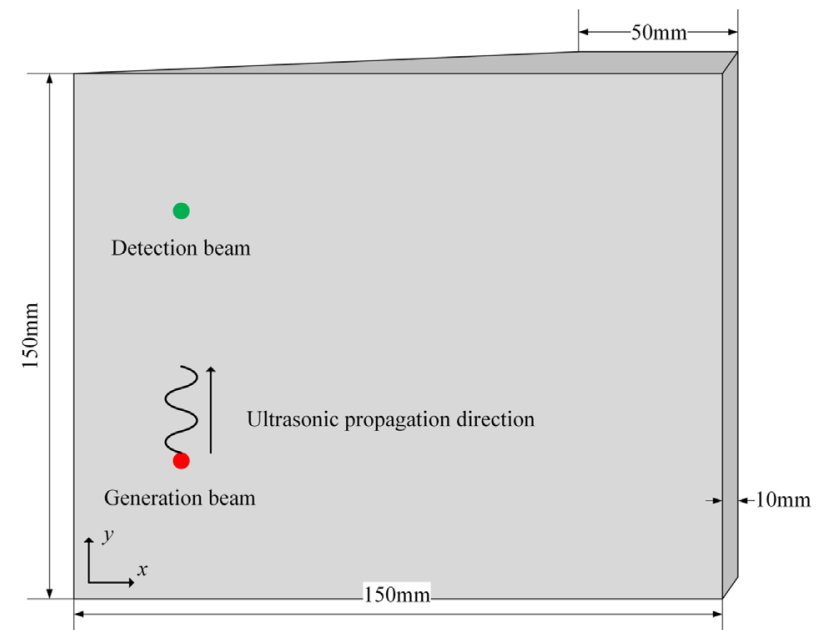

Figure 4. Schematic diagram of the wedge aluminum block.

diameter spot. The interferometer used an array receiver to improve detection sensitivity, while the out-of-plane and in-plane displacements were obtained simultaneously [16]. It may be noted that high sensitivity can be achieved even on a slightly rough surface because of the photorefractive effect [17]. A digital oscilloscope (Tektronix DPO 4054) was utilized to capture the output signal of interferometer, which was then averaged 64 times to increase the SNR. To determine what type of wave is measured, the wedge aluminum block was moved along the $x$ direction to change the testing thickness. The Lamb wave (highly dispersive A0 mode) [18] could be detected when the thickness was $1 \mathrm{~mm}$, as shown in Figure 5a.

The Lamb wave will approach the Rayleigh wave as the thickness of sample increases. As may be seen in Figure 5b, when the thickness $(1.5 \mathrm{~mm})$ is bigger than the wavelength of the Rayleigh wave $(1.465 \mathrm{~mm})$, Lamb wave begins to convert to Rayleigh wave [19]. The Rayleigh wave becomes dominant and the Lamb waves can be ignored when the thickness reaches $5 \mathrm{~mm}$.

\subsection{Experimental setup}

An aluminium block with dimensions $50 \times 90 \times 10 \mathrm{~mm}$ was used for the detection and reconstruction of surface defects as shown in Figure 6a. There were 4 holes (H1, $\mathrm{H} 2, \mathrm{H} 3, \mathrm{H} 4)$ at a surface depth of $3 \mathrm{~mm}$ whose diameters were $1 \mathrm{~mm}, 1.5 \mathrm{~mm}, 2 \mathrm{~mm}$ and $2.5 \mathrm{~mm}$ respectively. The experiment arrangement was the same as Figure 3 . The Nd:YAG laser was stationary during the experiment, while the sample and the interferometer were moved sequentially.

Regarding this particular experimental arrangement, the number of array elements was $N=20$ with a space of $1 \mathrm{~mm}$ ranging from $10 \mathrm{~mm}$ to $30 \mathrm{~mm}$ along the $x$ axis, as illustrated in Figure 6b. In addition, the center coordinates of the four holes were $(8,9.5)(16,9.5)(24,9.5)$ and $(32,9.5)$ respectively. In addition, separation between adjacent features must not less than a wavelength, or strong overlapping may appear in the TFM reconstruction [20]. 


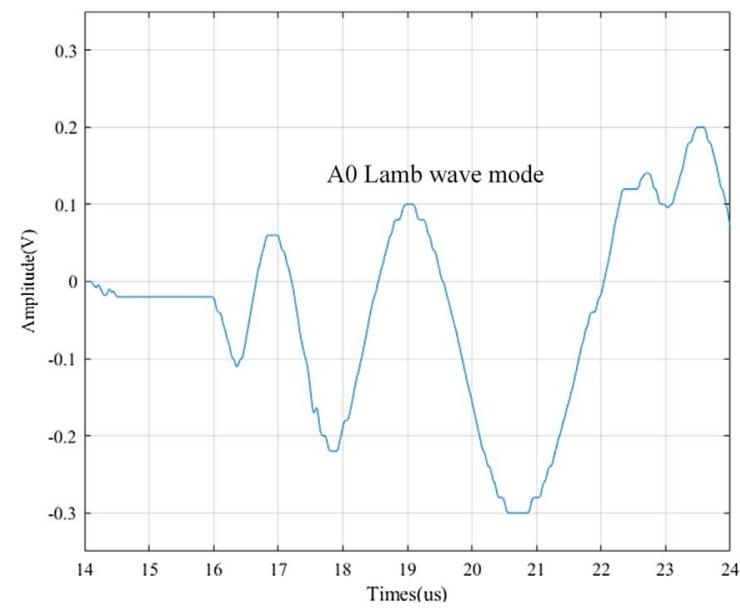

(a)

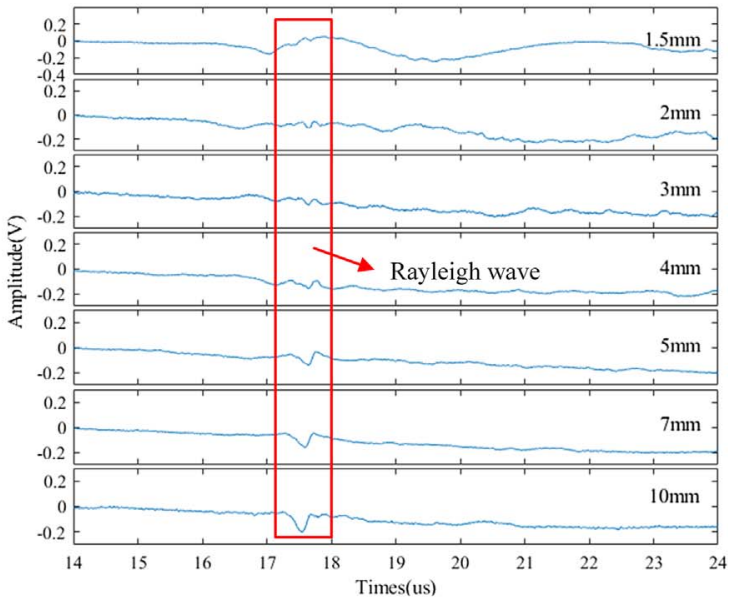

(b)

Figure 5. (a) The A0 Lamb wave mode detected at $1 \mathrm{~mm}$ thickness. (b) Measurement results at different thicknesses.

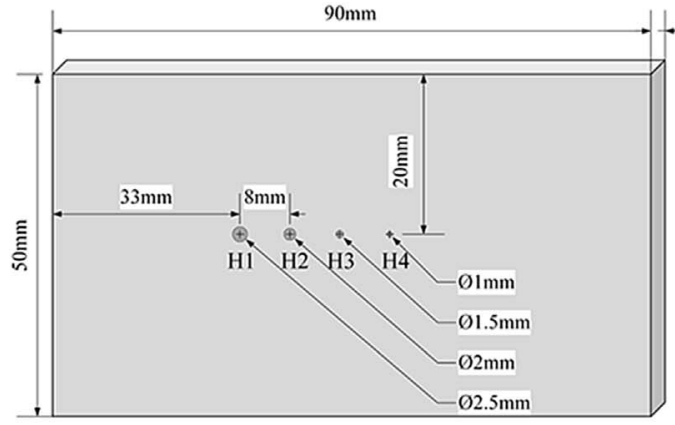

(a)

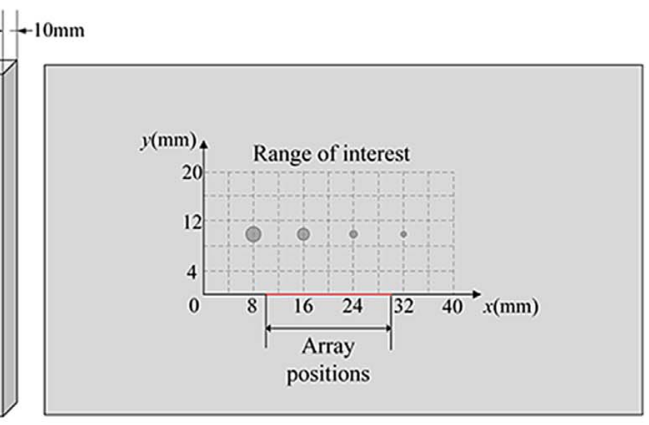

(b)

Figure 6. (a) The dimensional drawing of the aluminium block with four holes whose diameters range from $1 \mathrm{~mm}$ to $2.5 \mathrm{~mm}$. (b) Schematic diagram with the range of interest and array positions.

\subsection{Results}

The center frequency of the Rayleigh waves generated by the laser was $2 \mathrm{MHz}$ and the bandwidth of the selected filter was 1-4 MHz. The separation of the four holes is far greater than one wavelength, so defects can be well identified. Figure 7a shows a typical filtered A-scan waveform generated and detected at $x=16 \mathrm{~mm}$. The velocities of Rayleigh and longitudinal waves in aluminium are $2930 \mathrm{~m} / \mathrm{s}$ and $6100 \mathrm{~m} / \mathrm{s}$ respectively. It may be seen in this figure that the longitudinal wave is reflected from the bottom of the aluminium block and the Rayleigh waves are identified as the scattered signal after encountering four holes. The SNR of the reflected waves is significantly low. At the same time, the small size of these defects (i.e. holes), diffraction and scattering effects will occur. Part of the wave will mode-convert into bulk waves (longitudinal or shear waves) [21]. Figure $7 \mathrm{~b}$ gives the B-scan result using a part of the out-of-plane signals $S_{j j}(j=1,2 \ldots N)$. As expected, it is difficult to distinguish the surface defects from the B-scan image with poor lateral resolution which is mainly determined by the scanning step.
Figures $8 \mathrm{a}$ and $8 \mathrm{~b}$ show the results of SAFT and FMC/ TFM reconstruction. These two methods achieve improvements over B-scan technology including higher lateral resolution and larger imaging range. Moreover, SAFT and B-scans use the same data the former can significantly enhance the contrast of the defects boundary and reduce the interference of the longitudinal wave (bottom reflection). SAFT and FMC/TFM images can clearly show the location of four holes, though $\mathrm{H} 1$ and $\mathrm{H} 4$ are out of the scanning range. However, the SAFT image has relatively high background noise compared to FMC/TFM especially in the range from $0 \mathrm{~mm}$ to $5 \mathrm{~mm}$. The reason is the number of signals $\left(N^{2}\right)$ utilized in the $\mathrm{FMC} / \mathrm{TFM}$ process as opposed to $\operatorname{SAFT}(N)$. As a result, FMC/TFM improves imaging quality at the cost of measurement time.

In order to avoid the effects of the bottom-reflected longitudinal wave and the ultrasonic wave source on the results, the unwanted signals can be identified and removed based on the propagation time and pulse width [13]. The resulting SAFT and FMC/TFM images are presented in Figures 9a and 9b. The black dotted line marks the actual position of the holes. It may be seen from Figures $9 \mathrm{a}$ and $9 \mathrm{~b}$, 


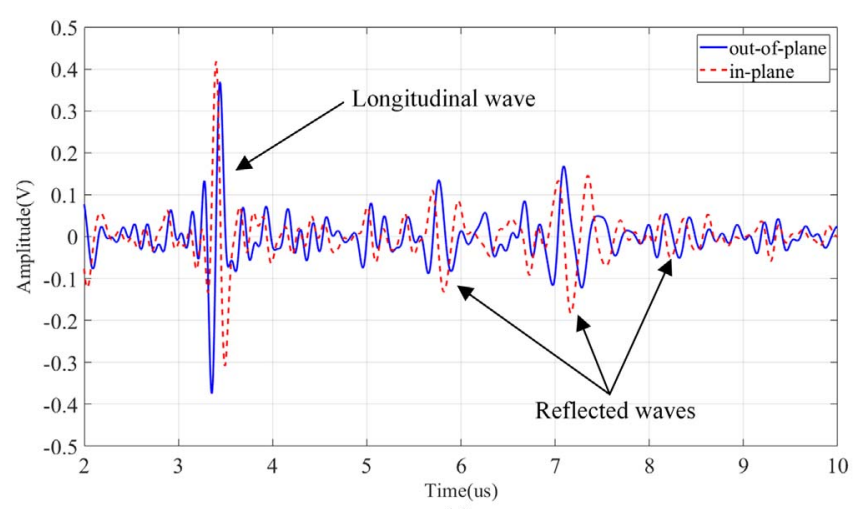

(a)

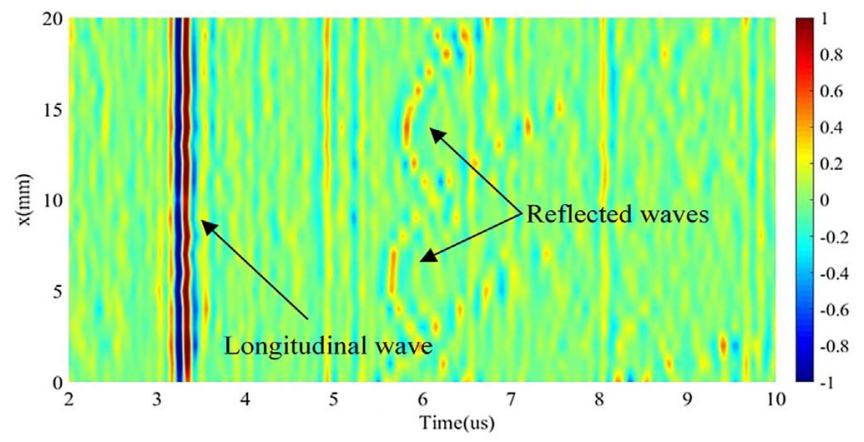

(b)

Figure 7. (a) A typical filtered A-scan signal acquired at $x=16 \mathrm{~mm}$. (b) B-scan results part of the out-of-plane signals $S_{j j}(j=1,2, \ldots, N)$.

that both imaging results of the two methods agree well at the actual defects boundaries. However, the SAFT imaging quality is relatively poor, and the defects are over-shadowed by noise. The maximum of the projection of the SAFT and FMC/TFM along the $x$ direction are shown in Figures $9 \mathrm{c}$ and $9 \mathrm{~d}$, which shows FMC/TFM technology can better suppress the background noise.

In this study, SNR is used as a metric to compare the performance of imaging algorithms, which is defined as:

$$
A_{\mathrm{SNR}}=20 \log _{10}\left(\frac{\operatorname{abs}\left(A_{p}\right)}{A_{R}}\right),
$$

where $A_{p}$ is the peak amplitude of the defect image and $A_{R}$ is the root mean square value of the result. The SNR values of different methods are listed in Table 1.

As may be seen in Table 1, B-scan technology can only image $\mathrm{H} 2$ and $\mathrm{H} 3$ in the scanning range and it is difficult to locate defects with low SNR, while SAFT and FMC/TFM technology allow for larger area testing with high SNR. Furthermore, FMC/TFM technology can greatly enhance the SNR than the other techniques.

\section{Discussion}

Surface defects like small holes were fabricated and used to verify the FMC/TFM technology. It has been shown that this detecting mechanism can improve the imaging
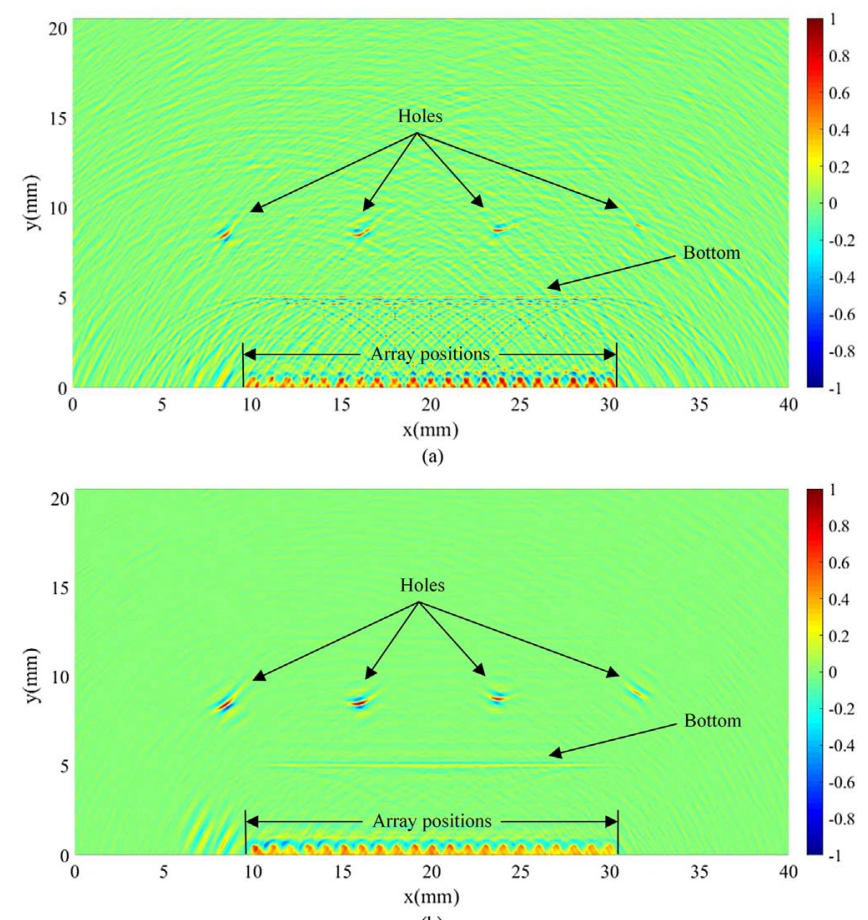

(b)

Figure 8. (a) The image of SAFT using the same data as B-scan. (b) The image of FMC/TFM reconstruction.

quality compared to SAFT and B-scan technologies. Although this method can significantly improve the SNR and successfully identify the surface defect boundary, FMC/TFM technology needs $N \times N$ points data acquisition instead of $N$ points for SAFT and B-scan technologies, which consequently increases the scanning time. To overcome this, a pulsed laser with higher repetition rates as well as a high-speed scanning mechanism is required. In addition, the appropriate array spacing should also be taken into consideration, which determines the directivity of the array.

The directivity pattern is the same as the optical fiber phased array, which is given as [22, 23]:

$$
O\left(\theta, \theta_{N}\right)=O_{1}(\theta) O_{N}\left(\theta, \theta_{N}\right)
$$

where $O_{1}(\theta)$ is the directivity of a single element, $O_{N}\left(\theta, \theta_{N}\right)$ is the directivity of array elements, which is given by:

$$
O_{N}\left(\theta, \theta_{N}\right)=\frac{\sin (N p)}{N \sin (p)}
$$

and

$$
p=\frac{\pi a\left(\sin (\theta)-\sin \left(\theta_{N}\right)\right)}{\lambda}
$$

where $N$ is the number of array elements, $a$ is array spacing, $\theta_{N}$ is the steering angle, $\theta$ is the angle between observation direction and $x$ axis, $\lambda$ is wavelength of Rayleigh wave. In this study, $\theta_{N}=\pi / 2, \lambda=1.465 \mathrm{~mm}$ and $a=1 \mathrm{~mm}(<\lambda)$. Rayleigh waves generated by point 


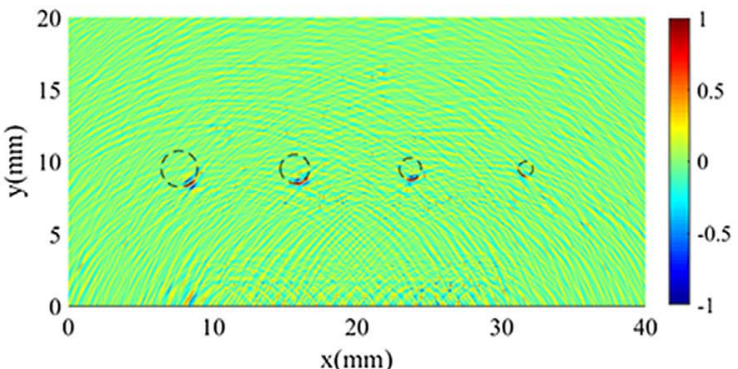

(a)

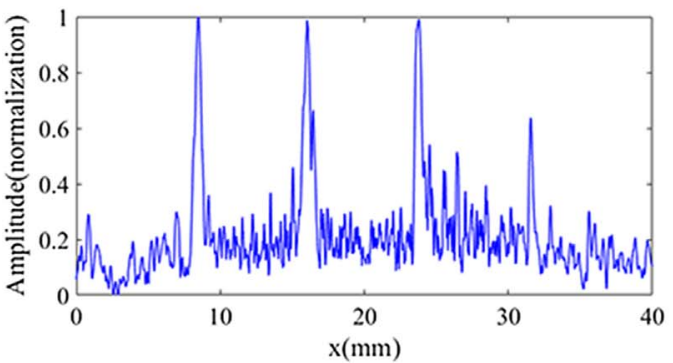

(c)

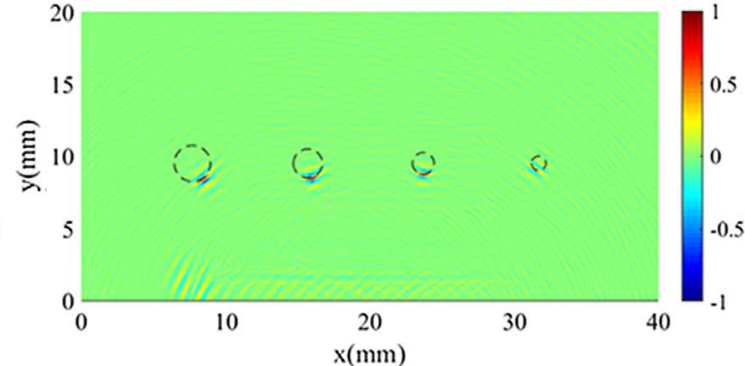

(b)

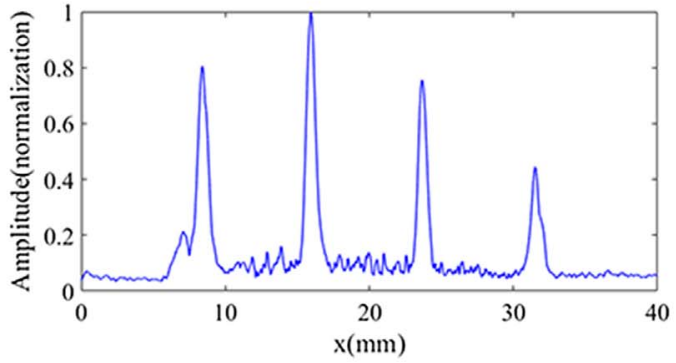

(d)

Figure 9. (a) The SAFT image after removing unwanted signals. (b) The FMC/TFM image after removing unwanted signals. (c) The maximum of the projection of SAFT along $x$ direction. (d) The maximum of the projection of the FMC/TFM along $x$ direction.

Table 1. The SNR values of different methods.

\begin{tabular}{lcccc}
\hline Technology & \multicolumn{4}{c}{ SNR $(\mathrm{dB})$} \\
\cline { 2 - 5 } & $H 1$ & $H 2$ & $H 3$ & $H 4$ \\
\hline B-scan & - & 11.8 & 13.7 & - \\
SAFT & 19.6 & 19.5 & 19.6 & 16.6 \\
FMC/TFM & 28.9 & 30.8 & 28.4 & 23.8 \\
\hline
\end{tabular}

sources are omnidirectional, so the directivity of a single element $O_{1}(\theta)=1$. The directivity pattern of this study is shown in Figure 10 (blue line), the results shows that the method of array can acquire improved directivity of the Rayleigh waves. With the purpose of obtaining a well-directed beam profile, the main lobe width should be minimized while suppressing the grating lobes. The lobe width can be considered as the distance between zero crossings $O_{N}\left(\theta, \theta_{N}\right)=0$ [24], so equations (5) and (6) can be written as:

$$
N a=\frac{n \lambda}{\sin (\theta)-1},(n= \pm 1, \pm 2 \cdots)
$$

As can be concluded from equation (7) that the lobe width decreases as the $N a$ increases, so the maximum spacing $a$ can not only improve the scanning speed but also strengthen the directivity of the sound beam. However, when $a=1.465 \mathrm{~mm}(=\lambda)$ and $\theta$ ranging from 0 to $\pi$, the grating lobes will appear and effect the imaging quality as shown in Figure 10, the red line and black line represent $a=1.465 \mathrm{~mm}(=\lambda), a=2 \mathrm{~mm}(>\lambda)$, respectively. In order to optimize the directivity of the array elements, the array spacing with $a=\lambda$ may be the best choice.

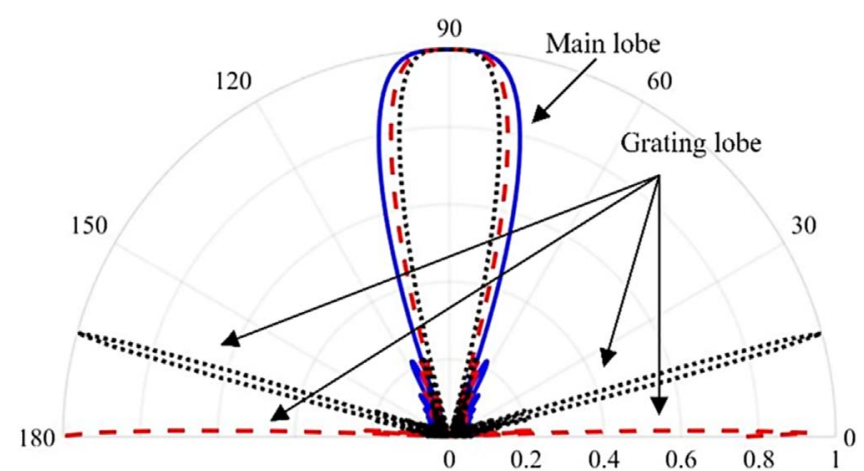

Figure 10. The directivity pattern of laser-induced ultrasonic array. The blue line, red line, black line represent $a=1 \mathrm{~mm}$ $(<\lambda), a=1.465 \mathrm{~mm}(=\lambda), a=2 \mathrm{~mm}(>\lambda)$, respectively.

\section{Conclusions}

Surface defects have various shapes and patterns, such as holes and cracks. Further work will focus on the experimentation of different defects regarding sizes and shapes. In addition, this technology will be experimented on the detection of surface defects for objects in harsh environments especially under high temperature and pressure, such as non-destructive testing of high-temperature pipelines.

In conclusion, this study presented a method to locate and image surface defects using full matrix capture and total focusing method based on laser-induced ultrasonic non-destructive testing. As opposed to traditional array ultrasonic surface defect detection, laser-induced ultrasound is a non-contact technology does not require wedgecoupling that avoids attenuation of high frequency acoustic 
signals and can detect complex structures in many harsh environments. Additionally, the FMC/TFM technology is applied in characterizing surface cracks without complex delay controls. Similar to SAFT, this technology utilizes synthetic apertures to improve the resolution and SNR of detection. As an example, an aluminium block with 4 holes at a depth of $3 \mathrm{~mm}$ with diameters ranging from $1 \mathrm{~mm}$ to $2.5 \mathrm{~mm}$ is used for testing. Compared to B-scan and SAFT, FMC/TFM has lower background noise and higher imaging contrast. In order to reduce the scanning time and optimize the directivity of array elements, the array spacing has been taken into consideration. When an array spacing of $a=\lambda$ the main lobe width should be minimized without doing so for the grating lobes. This method offers significant improvement and thus potential over existing methods for non-contact testing.

\section{Conflict of interest}

The authors declare that they do not have any conflict of interest.

\section{Acknowledgments}

The authors would like to acknowledge the funding and support of the National Key R\&D Program of China (2017YFF0206302), National Natural Science Foundation of China (NSFC) $(11904347,51805506)$, China Postdoctoral Science Foundation (2018M630192, 2019T120125) and Natural Science Foundation of Heilongjiang Province of China (LH2021E060).

\section{References}

1. R.S. Edwards, S. Dixon, X. Jian: Characterisation of defects in the railhead using ultrasonic surface waves. NDT \& E International 39 (2006) 468-475.

2.K.A. Tiwari, R. Raisutis, V. Samaitis: Hybrid signal processing technique to improve the defect estimation in ultrasonic non-destructive testing of composite structures. Sensors 17 (2017) 2858-2878.

3.D.A. Cook, Y.H. Berthelot: Detection of small surfacebreaking fatigue cracks in steel using scattering of Rayleigh waves. NDT \& E International 34 (2001) 483-492.

4. M. Duqennoy, M. Ouaftouh, M. Ourak: Determination of stresses in aluminium alloy using optical detection of Rayleigh waves. Ultrasonics 37 (1999) 365-372.

5. C.B. Thring, Y. Fan, R.S. Edwards: Focused Rayleigh wave EMAT for characterisation of surface-breaking defects. NDT \& E International 81 (2016) 20-27.

6. Y. Shi, Z. Shen, X. Ni, L. Jian, J. Guan: Ultrasonic phased array with surface acoustic wave for imaging cracks. AIP Advances 7 (2017) 065214.

7. C. Hoyle, M. Sutcliffe, P. Charlton, S. Mosey: Large-area surface imaging methods using ultrasonic Rayleigh waves, phased array and full matrix capture for non-destructive testing. Insight - Non-Destructive Testing and Condition Monitoring 61 (2019) 367-379.
8. M. Ducousso, F. Reverdy: Real-time imaging of microcracks on metallic surface using total focusing method and plane wave imaging with Rayleigh waves. NDT \& E International 116 (2020) 102311.

9. A. Ouchi, Y. Ohara, K. Yamanaka: Subharmonic phased array for crack evaluation using surface acoustic wave. Japanese Journal of Applied Physics 54 (2015) 07HC05.

10. M.H. Nadal, C. Hubert, A.R. Oltra: High temperature shear modulus determination using a laser-ultrasonic surface acousticwave device. Journal of Applied Physics 106 (2009) 024906.

11. J. Simpson, K.V. Wijk, L. Adam, C. Smith: Laser ultrasonic measurements to estimate the elastic properties of rock samples under in situ conditions. Review of Scientific Instruments 90 (2019) 114503.

12. C. Pei, D. Yi, T. Liu, X. Kou, Z. Chen: Fully noncontact measurement of inner cracks in thick specimen with fiberphased-array laser ultrasonic technique, NDT \& E International 113 (2020) 102273.

13. J. Chen, J. Xiao, D. Lisevych, Z. Fan: Laser-Induced Full-Matrix Ultrasonic Imaging of Complex-Shaped Objects, IEEE Transactions on Ultrasonics Ferroelectrics and Frequency Control 66 (2019) 1514-1520.

14. T. Stratoudaki, M. Clark, P.D. Wilcox: Laser induced ultrasonic phased array using full matrix capture data acquisition and total focusing method. Optics Express 24 (2016) 21921-21938.

15. C. Fan, M. Caleap, M. Pan, B.W. Drinkwater: A comparison between ultrasonic array beamforming and super resolution imaging algorithms for non-destructive evaluation, Ultrasonics 54 (2014) 1842-1850.

16. T.E. Blum, K.V. Wijk, B. Pouet, A. Wartelle: Multicomponent wavefield characterization with a novel scanning laser interferometer. The Review of scientific instruments 81 (2010) 073101.

17.S. Choi, H. Seo, K.Y. Jhang: Noncontact evaluation of acoustic nonlinearity of a laser-generated surface wave in a plastically deformed aluminum alloy. Research in Nondestructive Evaluation 26 (2015) 13-22.

18. C. Edwards, T. Stratoudaki, S. Dixon: Laser generated Rayleigh and lamb waves. American Institute of Physics AIP 284 (2002) 284-291.

19. R. Tao, W.B. Wang, J.T. Luo: Thin film flexible/bendable acoustic wave devices: Evolution, hybridization and decoupling of multiple acoustic wave modes. Surface and Coatings Technology 357 (2018) 73901.

20. N. Laroche, S. Bourguignon, E. Carcreff, J. Idier, A. Duclos: An inverse approach for ultrasonic imaging from full matrix capture data. Application to resolution enhancement in NDT. IEEE Transactions on Ultrasonics Ferroelectrics and Frequency Control 62 (2020) 1877-1887.

21. Z. Zhou, K. Zhang, J. Zhou, G. Sun, J. Wang: Application of laser ultrasonic technique for non-contact detection of structural surface-breaking cracks. Optics \& Laser Technology 73 (2015) 173-178.

22. J. Yang, N. DeRidder, C. Ume, J. Jarzynski: Non-contact optical fibre phased array generation of ultrasound for nondestructive evaluation of materials and processes. Ultrasonics 31 (1993) 387-394.

23. A.J.A. Bruinsma, J.A. Vogel: Ultrasonic noncontact inspection system with optical fiber methods. Applied Optics 27 (1988) 4690-4695.

24. S.C. Wooh, Y. Shi: Optimum beam steering of linear phased arrays. Wave Motion 29 (1999) 245-265. 